\title{
Mortality in Barrett's oesophagus: results from a population based study
}

\author{
L A Anderson, L J Murray, S J Murphy, D A Fitzpatrick, B T Johnston, R G P Watson, \\ P McCarron, A T Gavin
}

Gut 2003;52:1081-1084

\begin{abstract}
Background: Patients with Barrett's oesophagus have an increased risk of oesophageal adenocarcinoma but this cancer only accounts for a small proportion of deaths in these patients. Other causes of death are reportedly raised in this group. We examined cause specific mortality among individuals in a population based Barrett's oesophagus register. Methods: We constructed a register of all patients diagnosed with columnar mucosa (including specialised intestinal metaplasia) of the oesophagus within Northern Ireland between 1993 and 1999. Deaths occurring within this cohort until 31 December 2000 were identified and mortality rates were compared with the general population. Results: Overall mortality was not raised in Barrett's patients. During 7413 person years of follow up in 2373 patients there were 253 deaths (standardised mortality ratio (SMR) 96 (95\% confidence interval (CI) 84-107)). Mortality from oesophageal cancer was raised in patients with specialised intestinal metaplasia (SMR $774(95 \% \mathrm{Cl} 317-1231)$ ) but only $4.7 \%$ of patients died from this cancer. Mortality from stroke (SMR 65 (95\% Cl 37-93)) was significantly lower than the general population while mortality from non-cancerous digestive system diseases was significantly higher (SMR 211 (95\% Cl 111311 )). Mortality rates from all other causes were similar to those of the general population.

Conclusions: This study demonstrates that the overall mortality rate in patients with Barrett's oesophagus is closely similar to that of the general population. Oesophageal cancer mortality was raised but is an uncommon cause of death in these patients who also appear to have a reduced risk of death from stroke.
\end{abstract}

See end of article for authors' affiliations

Correspondence to: L A Anderson, Northern Ireland Cancer Registry, Department of Epidemiology and Public Health, The Queen's University Belfast, Mulhouse Building, Belfast BT12 6BJ, UK;

l.anderson@qub.ac.uk

Accepted for publication 20 February 2003

B arrett's oesophagus (BO) is a premalignant condition of the distal oesophagus where the normal squamous epithelium is replaced by columnar epithelium. Previous studies have shown that the risk of developing oesophageal adenocarcinoma (OAC) which is increasing in Western Europe $^{1-4}$ and in the USA, is much higher in patients with BO than in the general population. ${ }^{67}$ However, most patients with $\mathrm{BO}$ do not develop $\mathrm{OAC}^{7}$ and the mortality rate from oesophageal cancer has been reported to account for less than $10 \%$ of all deaths in these patients. ${ }^{8-10}$

Conflicting evidence has emerged concerning the overall mortality rates of patients with BO. In 1989 van der Veen and colleagues ${ }^{11}$ reported the follow up of 166 patients with BO over 4.4 years and found that survival was not different to an age and sex matched population. In 1996 however van der Burgh and coworkers" re-evaluated this group of patients, with a mean follow up of 9.3 years, and reported an excess mortality of $50 \%$ when death rates were compared with those of an age and sex matched control population. More recently, Eckardt and colleagues ${ }^{8}$ reported that the estimated 10 year survival of patients with long segment BO was similar to that of the general population. None of these studies was population based.

Sontag and colleagues ${ }^{12}$ reported an increased incidence of colonic tumours in patients with $\mathrm{BO}$ however; more recent studies $^{13} 14$ have found there to be no association between colorectal tumours and BO.

The present study uses a population based register to examine overall and cause specific mortality among patients diagnosed with BO.

\section{METHODS}

This is a follow up study of a population based cohort of individuals with $\mathrm{BO}$. The cohort comprises all adults identified

Abbreviations: $B O$, Barrett's oesophagus; OAC, oesophageal adenocarcinoma; NI, Northern Ireland; OGJ, oesophagogastric junction; SIM, specialised intestinal metaplasia; SMR, standardised mortality ratio. 
Table 1 Sex distribution, age, and SIM status in patients with Barret's oesophagus

\begin{tabular}{|c|c|c|c|c|}
\hline & \multicolumn{2}{|l|}{ Male } & \multicolumn{2}{|l|}{ Female } \\
\hline & n (\%) & Age $(y)^{*}$ & n (\%) & Age $(y)^{*}$ \\
\hline All patients & 1380 & $58.2(16-98)$ & 993 & $63.9(16-95)$ \\
\hline SIM present & $819(59.4)$ & $60.0(16-98)$ & $473(47.6)$ & $66.7(20-94)$ \\
\hline SIM absent & 257 (18.6) & $53.9(17-87)$ & $243(24.5)$ & 58.0 (16-92) \\
\hline SIM unknown & $304(22.0)$ & $57.1(19-90)$ & $277(27.9)$ & 64.3 (19-95) \\
\hline
\end{tabular}

study, BO was defined as the presence of columnar metaplasia in the oesophagus as noted on the pathology report form, irrespective of whether it was stated in the clinical summary that Barrett's mucosa was seen or suspected by the endoscopist. Only those biopsies with columnar metaplasia of the oesophagus were included and although it is possible that some of the biopsies were taken from an unrecognised hiatus hernia, the clinician believed the specimen to be from the distal oesophagus at endoscopy. All oesophageal biopsies with BO were further subdivided according to whether the pathologist specifically stated that specialised intestinal metaplasia (SIM) was present or absent in the biopsy specimen. If an oesophageal biopsy showed evidence of malignancy it was excluded from the study.

\section{Identification and classification of individual patients}

Many patients in the cohort had oesophageal biopsies taken on more than one occasion. Individual patients were identified within the data set by matching on surname, forename, and date of birth (and hospital numbers when they were available). The date of the earliest biopsy showing BO was taken as the date of entry into the cohort. Patients (rather than biopsies) were classified according to whether any of the oesophageal biopsies that had been taken showed SIM. If any of their biopsies showed SIM the patient was classified as having BO with SIM. If in all of the biopsies the pathologist had specifically stated that SIM was not present the patient was classified as having BO without SIM. Other patients were classified as having BO with SIM status unknown.

\section{Follow up}

Deaths among the cohort were identified between 1993 and 2000 inclusive, by matching each patient with death files from the General Registrar Office using the patient's surname, forename, and date of birth. In addition, possible matches, where there may have an error in entering the patient data onto the computer, were detected and checked manually by trained data abstractors. The files contain information on all the deaths that occur within NI, along with the causes of death and the date death occurred. For the purposes of analysis, only the primary cause of death was considered.

\section{Statistical analysis}

All analyses were conducted using SPSS for Windows. ${ }^{15}$ Patients diagnosed between 1993 and 1995 may have had a diagnosis of $\mathrm{BO}$ prior to entering into the cohort. In order to avoid inclusion of prevalent cases of $\mathrm{BO}$ we excluded this group from all analyses. Person years of follow up were calculated for each patient from the date of entry into the cohort (1995-1999) until the date of death or 31 December 2000, whichever was earlier. Standardised mortality ratios (SMRs) were calculated by comparing the observed number of deaths within the cohort to the expected number of deaths. The expected number of deaths were calculated by applying NI age specific cancer mortality rates to the cohort person years of follow up. SMRs were expressed as percentages (NI average 100 ) and the corresponding 95\% confidence intervals (CIs) were estimated.

\section{RESULTS}

Pathologists within NI reported a total of 15670 oesophageal biopsies performed between 1993 and 1999; 4955 (from 2969 patients) of these met the inclusion criteria for BO. There were 2373 patients diagnosed with BO between 1995 and 1999, $1380(58 \%)$ of whom were male (table 1). Males were on average younger than females at diagnosis: 58.2 years versus 63.9 years $(p<0.001)$. The annual rate of diagnosis of BO between 1993 and 1999 in NI was 4 per 1000 males and 2 per 1000 females.

Cases were followed up for up to six years, with the average follow up period being 3.1 years and the total person years of follow up 7413 years. During this period 253 (10.7\%) patients died; $139(55 \%)$ of these were men and 114 (45\%) were women. Mean age at death was 75.2 years, with men on average dying younger than women: 72.7 years versus 78.3 years $(\mathrm{t}=3.37, \mathrm{p}<0.001)$.

Overall, patients with $\mathrm{BO}$ had a similar mortality rate to that of the NI population (SMR 96 (95\% CI 84-107)) (table 2). Cancer accounted for 69 deaths (27.3\%) within the cohort but the SMR for cancer was similar to that of the NI population (SMR 122 (95\% CI 93-151)). Similar findings were observed when the analysis included only those patients with a diagnosis of SIM and when males and females were analysed separately (data not shown).

Oesophageal cancer accounted for $12(4.7 \%)$ of the deaths within the cohort (10 male, two female). This was significantly higher than the oesophageal cancer mortality rate expected in the NI population (2 expected, SMR 518 (95\% CI 225-812)). Males were found to have a significantly higher mortality rate from oesophageal cancer (SMR 604 (95\% CI 229-978)) than the NI population. This rate was also higher in females but to a lesser extent and was not statistically significant (SMR 235 (95\% CI 0-562)). When patients with BO were divided into three groups according to the presence of SIM it was found that only patients with SIM (11 patients) had a significantly increased oesophageal cancer mortality rate (SMR 774 (95\% CI 317-1231)). Mortality rates from all other cancers, including colorectal cancer (SMR 139 (95\% CI 53-225)), were not significantly different to those of the general population.

Non-malignant diseases of the oesophagus, stomach, and duodenum accounted for 17 deaths. These patients died from complications of oesophageal, gastric, or duodenal ulcers, including perforation and gastrointestinal haemorrhage. The mortality rate from these diseases in Barrett's patients was significantly higher than in the general population (SMR 211 (95\% CI 111-311)).

The death rate from stroke among Barrett's patients was significantly lower than in the NI population (SMR 65 (95\% CI 37-93)). This was seen in both males and females although the decreased ratio in women was not statistically significant. When patients with SIM were analysed separately, SMR decreased further to 50 (95\% CI 19-81). All other causes of death within the $\mathrm{BO}$ cohort had similar mortality rates to the general NI population. 
Table 2 Association between Barrett's oesophagus and SIM, and cause specific mortality

\begin{tabular}{|c|c|c|c|c|c|c|}
\hline \multirow[b]{2}{*}{ Cause of death (ICD9.0 codes) } & \multicolumn{2}{|c|}{$\begin{array}{l}\text { Observed mortality } \\
\text { (No of deaths) }\end{array}$} & \multicolumn{2}{|c|}{$\begin{array}{l}\text { Expected mortality } \\
\text { (No of deaths) }\end{array}$} & \multicolumn{2}{|l|}{ SMR $(95 \% \mathrm{CI})$} \\
\hline & BO & SIM & BO & SIM & BO & SIM \\
\hline All causes (1-999) & 253 & 149 & 265 & 163 & $96(84-107)$ & 91 (77-106) \\
\hline All cancers (140-239) & 69 & 41 & 56 & 35 & $122(93-151)$ & $118(82-155)$ \\
\hline Oesophageal cancer (150) & 12 & 11 & 2 & 1 & $518(225-812)^{*}$ & ${ }^{774}(317-1231)^{*}$ \\
\hline Stomach cancer (151) & 3 & 2 & 3 & 2 & 97 (0-207) & $106(0-252)$ \\
\hline Colorectal cancer $(153,154)$ & 10 & 4 & 7 & 4 & $139(53-225)$ & $90(2-179)$ \\
\hline Lung cancer (162) & 13 & 8 & 12 & 7 & 107 (49-165) & 107 (33-181) \\
\hline Digestive system disease (520-579) & 17 & 10 & 8 & 5 & $211(111-311)^{*}$ & 202 (77-327) \\
\hline Ischaemic heart disease (410-414) & 60 & 37 & 66 & 41 & $91(68-113)$ & $90(61-119)$ \\
\hline Stroke $(430-438)$ & 21 & 10 & 32 & 20 & $65(37-93)^{*}$ & $50(19-81)^{*}$ \\
\hline Other circulatory disease $(390-409,415-429,439-459)$ & 16 & 11 & 21 & 13 & $74(38-111)$ & $83(34-132)$ \\
\hline Respiratory system disease (460-519) & 46 & 27 & 57 & 35 & 81 (57-104) & $76(48-105)$ \\
\hline Other causes $(1-139,240-389,580-999)$ & 24 & 13 & 23 & 14 & $103(62-145)$ & $91(42-141)$ \\
\hline
\end{tabular}

$\mathrm{BO}$, Barrett's oesophagus; SIM, specialised intestinal metaplasia; SMR, standardised mortality ratio; $95 \% \mathrm{Cl}, 95 \%$ confidence interval. ${ }^{*} \mathrm{p}<0.05$.

\section{DISCUSSION}

This is, to date, the largest study examining causes of mortality in patients with BO. All incident cases of $\mathrm{BO}$ within NI were identified during the study period. Emigration out of $\mathrm{NI}$ in people over 50 years is low, reducing problems associated with loss to follow up. Although long term follow up was not possible, the number of cases in this study (2373) far exceeds any of those carried out previously, with the total person years of follow up amounting to 7413 years. This and the population based nature of our study, which minimises selection biases, is likely to provide a robust estimate of mortality in patients with BO.

In accordance with the observations of other research groups ${ }^{811}{ }^{16}$ patients with BO had mortality rates similar to an age and sex matched control population. This conflicts with the findings of a smaller study by van der Burgh and colleagues ${ }^{9}$ who followed up 166 patients with $\mathrm{BO}$ over a mean period of 9.3 years ( 1440 person years). Differences in study design, in particular size and period of follow up, are likely to have resulted in this discrepant finding. It is also possible that mortality rates could have been underestimated in the current study, if some patients included in the cohort had a biopsy taken from an unrecognised hiatus hernia.

Oesophageal cancer accounted for only $4.7 \%$ of all deaths within the group, which is similar to other reports that only a small proportion of the total number of deaths, between $0 \%$ and $10 \%,{ }^{17}$ result from oesophageal cancer. However, oesophageal cancer mortality was significantly increased in the cohort when compared with the NI population. Since the first definition of BO in $1950,{ }^{18}$ it has become apparent that the presence of SIM confers a greater risk of developing OAC. ${ }^{19}{ }^{20}$ The findings of this study indicate that only patients with SIM have an increased mortality rate from oesophageal cancer, approximately eight times that of the normal population. Furthermore, males had more than twice the mortality rate from oesophageal cancer than females. These findings suggest that surveillance programmes could be limited to patients with SIM $^{1720}$ and targeted towards men. Although avoided mortality was not quantified and needs to be considered, the relatively low mortality from oesophageal cancer, even in these patients, calls into question the cost effectiveness of surveillance programmes that cannot be targeted at patients with a high risk of cancer.

Overall cancer mortality was not significantly raised in the cohort of Barrett's patients. This is in keeping with the observation of Drewitz and colleagues ${ }^{21}$ who noted that $34 \%$ of the deaths among Barrett's patients were from cancer, a rate similar to the percentage of deaths from cancer in males in the USA. Our study showed no significant increase in the mortality rate from colorectal cancer in comparison with the general population, indicating that previous suggestions of surveillance for colorectal cancer ${ }^{122}$ are unwarranted.

Increased mortality rates from diseases of the digestive system, especially peptic ulcer disease, were observed. Diagnosis of BO in such patients may have been an incidental finding during endoscopic procedures to investigate the conditions, which led to their deaths. Alternatively, increased acid secretion or decreased mucosal defence may be underlying pathophysiological mechanisms common to both $\mathrm{BO}$ and peptic ulcer disease.

Patients with BO (especially those with SIM) were found to have a significantly lower mortality rate from stroke than those in the NI population, suggesting a possible association between $\mathrm{BO}$ and stroke mortality. Further work is required to confirm or refute this association. Surveillance of patients with $\mathrm{BO}$ may have attributed to a reduction in deaths from stroke due to investigation for other medical conditions at their follow up appointments, or the "worried well" may be more likely to undergo endoscopy and have incidental BO diagnosed. However, in both of these circumstances we would expect a reduction in overall mortality, which was not observed in this study. Perhaps individuals with low vascular tone, and hence low blood pressure and stroke risk, also have disturbed oesophageal motor function, allowing gastrooesophageal reflux and the development of $\mathrm{BO}$.

This study reinforces the findings of previous studies that oesophageal cancer is an uncommon cause of death in patients with $\mathrm{BO}$ and that the increased mortality rate from oesophageal cancer is confined to patients with SIM. Additionally, the study shows that the overall mortality rate in Barrett's patients is not increased when compared with that of the general population and indicates that these patients may have a reduced mortality rate from stroke.

\section{ACKNOWLEDGEMENTS}

The authors thank Colin Fox, Richard Middleton, Tom Wylie, and the Tumour Verification Offices of the Northern Ireland Cancer Registry for their assistance with the processing of the pathology records. Thanks are also due to the administrative, medical, and pathology staff of local Health Care Trusts and the staff of the Directorate of Information Services DHSSPS (NI) for assisting in the compilation of the Northern Ireland Barrett's Register.

LM, BJ, RW, and AG established the Northern Ireland Barrett's Register. LM developed the hypothesis under investigation. DF performed the statistical analysis. LA and LM drafted the manuscript. SM and PMCC edited subsequent drafts and all authors commented on the final draft. LM is the guarantor. 
The Northern Ireland Cancer Registry is funded by DHSSPS (NI). The Ulster Cancer Foundation provided part funding for the establishment of the Northern Ireland Barrett's Register.

\section{Authors' affiliations}

L A Anderson, L J Murray, S J Murphy, D A Fitzpatrick, P McCarron,

A T Gavin, Northern Ireland Cancer Registry, Department of

Epidemiology and Public Health, The Queen's University Belfast, UK

B T Johnston, The Royal Hospitals Trust, Belfast, UK

R G P Watson, Department of Medicine, The Queen's University Belfast, UK

\section{REFERENCES}

1 Hansson LE, Sparen P, Nyren O. Increasing incidence of both major histological types of esophageal carcinomas among men in Sweden. Int $J$ Cancer 1993:54:402-7.

2 Botterweck AAM, Schouten $L$, Volovics A, et al. Trends in incidence of adenocarcinoma of the oesophagus and gastric cardia in ten European countries. Int J Epidemiol 2000;29:645-54.

3 Powell J, McConkey CC. The rising trend in oesophageal

adenocarcinoma and gastric cardia. Eur J Cancer Prev 1992;1:265-9.

4 Moller $\mathbf{H}$. Incidence of cancer of oesophagus, cardia and stomach in Denmark. Eur J Cancer Prev 1992;1:159-64.

5 Blot WJ, McLaughlin JK. The changing epidemiology of esophageal cancer. Semin Oncol 1999;26(suppl 15):2-8.

6 Hameeteman W, Tytgat GNJ, Houthoff HJ, et al. Barrett's esophagus: development of dysplasia and adenocarcinoma. Gastroenterology 1989:96:1249-56

7 Spechler SJ, Robbins AH, Rubins HB, et al. Adenocarcinoma and Barrett's esophagus: an overrated risk? Gastroenterelogy 1984;87:927-33

8 Eckardt VF, Kanzler G, Bernhard G. Life expectancy and cancer risk in patients with Barrett's esophagus: a prospective controlled investigation. Am J Med 2001;111:33-7.
9 van der Burgh A, Dees J, Hop WCJ, et al. Oesophageal cancer is an uncommon cause of death in patients with Barrett's oesophagus. Gut 1996;39:5-8

10 Macdonald CE, Wicks AC, Playford RJ. Final results from 10 year cohort of patients undergoing surveillance for Barrett's oesophagus: observational study. BW 2000;321:1252-4.

11 van der Veen AH, Dees J, Blankensteijn JD, et al. Adenocarcinoma in Barrett's oesophagus: an overrated risk. Gut 1989;30:14-8.

12 Sontag SJ, Schnell TG, Chejfec G, et al. Barrett's oesophagus and colonic tumours. Lancet 1985;1:946-8.

13 Laitakari R, Laippala P, Isolauri J. Barrett's oesophagus is not a risk factor for colonic neoplasia: a case-control study. Ann Med 1995; 27:499-502.

14 Post AB, Achkar E, Carey WD. Prevalence of colonic neoplasia in patients with Barrett's oesophagus. Am J Gastroenterol 1993;88:877-80

15 SPSS for Windows, Rel. 11.0.1. Chicago: SPSS Inc, 2001.

16 Cameron AJ, OH BJ, Payne WS. The incidence of adenocarcinoma in columnar-lined (Barrett's) esophagus. N Engl J Med 1985;313:857-9.

17 Rana PS, Johnston DA. Incidence of adenocarcinoma and mortality in patients with Barrett's oesophagus diagnosed between 1976 and 1986 : implications for endoscopic surveillance. Dis Esophagus 2000;13:28-31.

18 Barrett NR. Chronic peptic ulcer of the oesophagus and "oesophagitis". Br J Surg 1950;38:175-82

19 Wolf C, Timmer R, Breumelhof R, et al. Columnar lined oesophagus and intestinal metaplaisa: current concepts. Eur J Gastroenterol Hepatol $1999 ; 11: 793-800$

20 McGarrity TJ. Barrett's oesophagus: the continuing conundrum. BM 2000;321:1238-9.

21 Drewitz DJ, Sampliner RE, Garewal HS. The incidence of adenocarcinoma in Barrett's esophagus: a prospective study of 170 patients followed 4.8 years. Am J Gastroenterol 1997;92:212-15.

22 Robertson DAF, Ayres RCS, Smith CL. Screening for colonic cancer in patients with Barrett's oesophagus. BMV 1989;298:650. 